\title{
Nano-scale, Ising-like Magnetic Domain Structures in Fe/NiO/Fe(100) Trilayers.
}

\author{
A.K. Schmid ${ }^{1}$, N. Rougemaille ${ }^{1}$, M. Portalupi ${ }^{2}$, A. Lanzara ${ }^{1,3}$, P. Biagioni $^{2}$, A. Brambilla ${ }^{2}$, M.

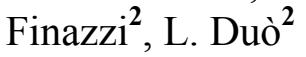 \\ ${ }^{1}$ National Center for Electron Microscopy, Lawrence Berkeley National Lab, Berkeley, CA 94720 \\ 2 Dipartimento di Fisica, Politecnico di Milano, Piazza L. da Vinci 32, 20133 Milano, Italy \\ ${ }^{3}$ Department of Physics, University of California at Berkeley, Berkeley, CA 94720
}

What determines the sizes and shapes magnetic domains can have at finite temperature? The question is important both fundamentally and because small/stable domains are potentially useful for data-storage and other applications. An interesting type of domain microstructures can be observed when ferromagnetic films interact with antiferromagnets. In the capping layer of $\mathrm{Fe} / \mathrm{NiO} / \mathrm{Fe}(100)$ trilayer structures, we find extremely delicate and convoluted magnetic domain patterns. The patterns include many domains that are topolocically unconnected ("bubbles") and narrow elongated domains ("channels"), and the domain wall widths are much smaller than what is observed in Fe layers grown on non-magnetic substrates. In fact, the widths of some bubble- and channel-domains are smaller than the widths of just the walls of domains we observe in our Fe/MgO(100) substrates!

Our structures were build on top of clean $\mathrm{MgO}(100)$ crystals, using in-situ preparation techniques. Freshly fabricated $\mathrm{NiO} / \mathrm{Fe} / \mathrm{MgO}(100)$ samples (thickness: $\mathrm{MgO}=$ bulk, $\mathrm{Fe} \approx$ hundreds $\mathrm{nm}, \mathrm{NiO} \approx 1$ $\mathrm{nm})$ were UHV-transferred into the specimen chamber of our spin-polarized low-energy electron microscope (SPLEEM), where we grew the top Fe layer (thickness $\approx 1 \mathrm{~nm}$ ) under conditions of very good vacuum and very low stray-magnetic field. We imaged the resulting, 'virgin', magnetic domain microstructure in the top Fe layer in-situ during and after deposition. When the thickness of the $\mathrm{NiO}$ spacer was small (of the order of $1 \mathrm{~nm}$ ), remarkable topology of the magnetic domains in the Fe capping layer results. Even though both the [010] and [001] direction are degenerate, in-plane, easymagnetization axes in these films, large regions of the samples are covered with intricate patterns of domains that are all magnetized along just one of the two easy axes. The domains are separated by $180^{\circ}$ domain walls and have complex, meandering structures. The widths of the domain walls are so small that, given the resolution of our images, we can only estimate an upper limit of $<20 \mathrm{~nm}$ wallwidth. This means the domain walls are one order of magnitude narrower than the size of $180^{\circ}$ walls we can observe in the epitaxial Fe films we use as substrates.

The topologies of the observed domain microstructures share many features with the predictions from two-dimensional, two-states Potts models (Ising-models) with only short-range interactions, which are sometimes invoked to describe magnetic systems with a single easy axis of magnetization. For example, unconnected topologies with domain size distributions that include very small bubbles and narrow channels, as well as spanning-domains that extend across the entire sample, are a natural feature of two-states systems [1]. What's puzzling is that, in principle, our system has four-fold symmetry, and one might have guessed that observed topologies should be closer to predictions from 4-states Potts models. (4-states models would predict compact domains and very rare bubbles or channels [1], this is clearly not our observation.) How can domains in the top Fe layer "choose" one of the two degenerate easy axes and "ignore" the other one? This can be understood as a result of the strong coupling of the ultrathin ferromagnetic top Fe-layer, through an antiferromagnetic spacer, to the much thicker ferromagnetic substrate [2]. The thick, ferromagnetic substrate has very large 
magnetic domains (typically mm-scale), magnetized along either [010] or [001] directions. The antiferromagnetic easy axis of $\mathrm{NiO}$ grown on top of such a domain latches onto the $\mathrm{Fe}$ magnetization direction via exchange coupling. As a result, antiferromagnetic domains in the $\mathrm{NiO}$ spacer scramble the coupling direction with respect to the subsequently deposited top Fe-layer, but conserve their axis with respect to the underlying domain in the Fe substrate. Exchange coupling to the NiO spacer is the origin of uniaxial, in-plane magnetic anisotropy in the top Fe-layer of our system. As shown in the figure, every large ferromagnetic domain in the Fe-substrate layer leads to a corresponding region in the top Fe-layer where we find two degenerate, anti-aligned magnetization directions.

The other puzzling feature in our observations is the extremely small width of domain walls in the top Fe-layer. Again, this can be understood as a consequence of exchange coupling between ferromagnetic and antiferromagnetic layers [2]. In pure ferromagnets, domain wall width is often relatively large, because it is governed by the competition between the stronger exchange interaction (favoring greater width) and normally relatively weak anisotropy (favoring smaller width). The situation can be very different at interfaces with antiferromagnets. In this case, frustrated exchange forces at interfaces also favor narrow domain walls, and can be far greater than anisotropy forces. This can lead to domain wall widths that are narrower than conventional domain walls by a substantial factor [2]. In light of potential data-storage applications, it is interesting to note that the stability of our observed nanodomains coincides with perfect remanence. Magneto-optic Kerr measurements [3] indicate that remanence of the films can be as large as saturation, which indicates that remanent single-domain states spanning the sample are possible, and one might envision to "write" individual, stable, "bubble"-nanodomains into such trilayer structures.

[1] Gary S. Grest and David J. Srolovitz, Phys. Rev. B30, 5150 (1984)

[2] A.I. Morosov and A.S. Sigov, Physics of the Solid State 46, 395 (2004)

[3] P. Biagioni, A. Brambilla, M. Portalupi, N. Rougemaille, A. K. Schmid, A. Lanzara, P.

Vavassori, M. Zani, M. Finazzi, L. Duò, F. Ciccacci, J. Magn. Magn. Mat., (in press, 2005)
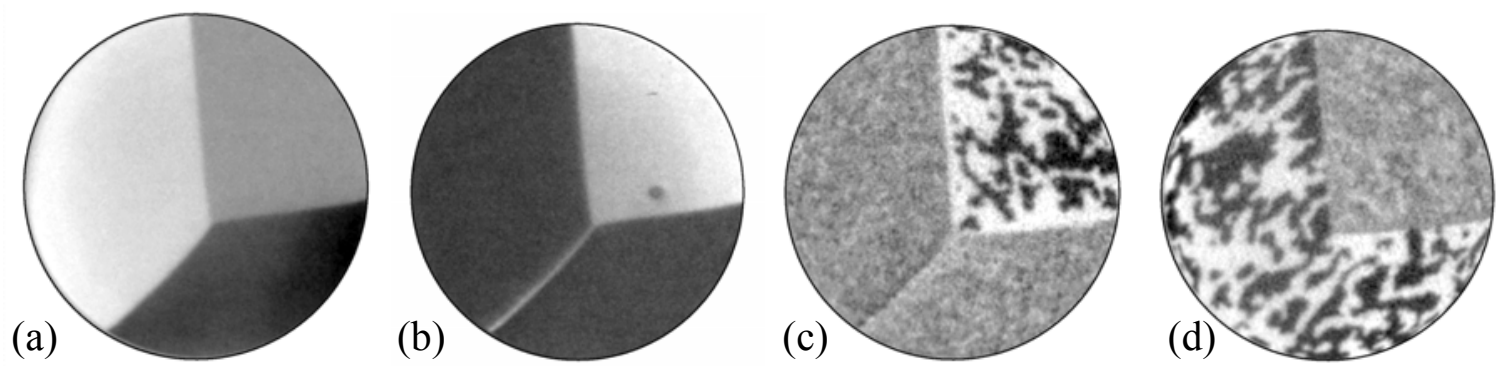

Figure: SPLEEM images (field of view $7 \mu$ ) in panels (a) and (b) (electron beam spin parallel [010] and [001], respectively) show magnetic domains in $\mathrm{Fe} / \mathrm{MgO}$ substrate prior to growth of trilayer structure. Large domains are magnetized along [010] or [001] easy axes. $180^{\circ}$ domain walls are $\approx$ $120 \mathrm{~nm}$ wide (see bright linear feature in lower part of b). Panels (c) and (d) show corresponding images of domain structures in the top Fe-layer after completion of the $\mathrm{Fe} / \mathrm{NiO} / \mathrm{Fe} / \mathrm{MgO}$ trilayer. Delicate, Ising-like domain structures include very small "bubble"- and "channel"-domains with width as small as $70 \mathrm{~nm}$. Width of $180^{\circ}$ domain walls is smaller than $20 \mathrm{~nm}$, below resolution of these images. 